\title{
Cross-talk between individual phenol soluble modulins in $S$. aureus biofilm formation
}

\author{
Masihuz Zaman ${ }^{1}$ and Maria Andreasen ${ }^{1 *}$
}

${ }^{1}$ Aarhus University, Department of Biomedicine, Wilhelm Meyers Allé 3, 8000 Aarhus, Denmark

*Correspondences should be addressed to MA (email: mariaj@biomed.au.dk) 


\begin{abstract}
The infective ability of the opportunistic pathogen Staphylococcus aureus is associated with biofilm mediated resistance to host immune response and even disinfectants and indeed $S$. aureus is recognized as the most frequent cause of biofilm associated infections. Phenol-soluble modulin (PSM) peptides serve various roles in pathogenicity while also comprising the structural scaffold of S. aureus biofilms through self-assembly into functional amyloids, but the role of the individual PSMs during biofilm formation remains poorly understood and the molecular pathways of PSM selfassembly have proved challenging to identify. Here, we show a high degree of cooperation between individual PSMs during the formation of functional amyloids in biofilm formation. The fast aggregating PSM $\alpha 3$ initiates the aggregation by forming unstable aggregates capable of seeding the formation of aggregates by other PSM peptides into the formation of stable amyloid structures. Using chemical kinetics along with spectroscopic techniques we dissect the molecular mechanism of aggregation of the individual peptides to show that PSM $\alpha 1$, PSM $\alpha 3$ and PSM $\beta 1$ display secondary nucleation whereas $\beta$ PSM2 aggregates through primary nucleation and elongation. Our findings suggest that the various PSMs have evolved to ensure fast and efficient biofilm formation through cooperation between individual peptides.
\end{abstract}

\title{
INTRODUCTION
}

Aggregated proteins in the form of functional amyloids are widespread in Nature [1]. In humans, functional amyloids assist in immunity, reproduction and hormone secretion [2]. However, in various bacterial strains they provide structural stability as the major protein component of the self-produced polymeric matrix in biofilms [3-6]. Functional amyloids increase the bacteria's ability towards a variety of environmental insults, increasing their persistence in the host as well as promoting resistance to antimicrobial drugs and the immune system [7-9]. The well-studied curli machinery in 
Escherichia coli [3], Fap system in Pseudomonas fluorescens [4], TasA system in Bacillus subtilis [5], along with phenol-soluble modulins (PSMs) in Staphylococcus aureus [6] are some of the major bacterial functional amyloid systems that have been reported so far.

For $S$. aureus biofilm formation PSMs have been recognized as a crucial factor. In their soluble monomeric form they hinder host immune response by recruiting, activating and lysing human neutrophils while also promoting biofilm dissociation [6]. However, self-assembly of PSMs into amyloid fibrils fortify the biofilm matrix to resist disassembly by mechanical stress and matrix degrading enzymes [10]. The genes encoding the core family of PSMs peptides are highly conserved and located in $p s m \alpha$ operon (PSM $\alpha 1-\mathrm{PSM} \alpha 4), p \operatorname{sm} \beta$ operon (PSM $\beta 1$ and PSM $\beta 2$ ) and the $\delta$-toxin is encoded within the coding sequence of RNAIII [11]. High expression of PSMas, 20 residues in length, increases virulence potential of methicillin-resistant S. aureus [12]. Moreover, PSM $\alpha 3$ the most cytotoxic and lytic PSM, enhances its toxicity to human cells upon fibrillation [13]. Despite lower concentrations, the larger PSM $\beta$ s, 44 residues in length, seem to have the most pronounced impact on biofilm structuring [14]. Despite the formation of functional amyloids in S. aureus by PSMs, many questions remain about the intrinsic molecular mechanism by which they self-assemble and what molecular events triggers the formation of fibrillar structure from their monomeric precursor peptide. Here, we apply a combination of chemical kinetic studies along with biophysical techniques to explore the relative importance of different microscopic steps involved in the mechanism of fibril formation of PSMs peptides.

\section{RESULTS}

\section{Chemical kinetics reveal different aggregation mechanisms for different PSMs}

To investigate the dominating mechanism of aggregation for the individual PSMs we used chemical kinetics to analyze the aggregation of all the seven individual PSM peptides under quiescent 
conditions. Recently, kinetic models of protein aggregation $[15,16]$ have been effectively applied to numerous model systems in biomolecular self-assembly [17-19]. Through these models, the aggregation kinetics ascertain the rates and reaction orders of the underlying molecular events, allowing for the determination of the dominating molecular mechanism of formation of new aggregates. Aggregation kinetics of all seven PSMs peptides (PSM $\alpha 1-4, \mathrm{PSM} \beta 1-2$ and $\delta$-toxin) was monitored using Thioflavin T (ThT) fluorescence intensity [20]. For PSM $\alpha 1$, PSM $\alpha 3$, PSM $\beta 1$ and PSM 32 reproducible aggregation curves were observed, Fig $1 \mathrm{a}-\mathrm{c}$ and $1 \mathrm{~g}$, while for the rest of the PSM peptides (PSM $\alpha 2$, PSM $\alpha 4$ and $\delta$-toxin) no reproducible aggregation was observed, Fig. S1a-c. An increase in ThT fluorescence is observed for PSMa4 although this was not sigmoidal in shape and also not reproducible, Fig. S1b. The timescale for the completion of aggregation differs significantly between the PSM ranging from $\sim 1 \mathrm{~h}$ for PSM $\alpha 3$ and up to $\sim 70 \mathrm{~h}$ for PSM $\alpha 1$. Furthermore, the aggregation of PSM $\beta 1$ was carried out at concentrations of $\mu \mathrm{g} / \mathrm{mL}$ compared to concentrations at $\mathrm{mg} / \mathrm{mL}$ for the other PSM peptides since at higher concentrations of PSM $\beta 1$ the lag-time during aggregation becomes monomer independent suggesting a saturation effect, Fig S1d.

To elucidate the dominating aggregation mechanism of PSM $\alpha 1, \operatorname{PSM} \alpha 3, \mathrm{PSM} \beta 1$ and PSM $\beta 2$, kinetic data were globally fitted at all monomeric concentrations concurrently by kinetic equations using the Amylofit interface (http://www.amylofit.ch.cam.ac.uk/fit) [21]. High quality global fits were achieved for all four peptides assuming a secondary nucleation mechanism for PSM $\alpha 1$, PSM $\alpha 3$ and PSM $\beta 1$, and a primary nucleation and elongation mechanism for PSM $\beta 2$. The presence of a single dominating aggregation mechanism for all four peptides is seen in the linear correlation between the half-time and the initial monomer concentration [21], Fig. S2a-d. In this simple nucleation-elongation (or linear self-assembly) model, the protein monomers form an initial nucleus with rate constant $\left(k_{n}\right)$ and reaction order $\left(n_{c}\right)$ which grow by elongation through the addition of monomers to the fibrils ends with rate constant $\left(k_{+}\right)$. The secondary nucleation model additionally involves nucleus formation 
catalyzed by existing aggregates. In this model system, $k$ act as a combined parameter that controls the proliferation through secondary pathways with secondary process rate constant $\left(k_{2}\right)$ and secondary pathway reaction order $\left(n_{2}\right)$ with respect to monomer [21]. Secondary nucleation dominated aggregation mechanisms have previously been reported for disease related amyloid fibrils, for example A $\beta$ peptides [17, 18], insulin [22], $\alpha$-synuclein [23, 24] and islet amyloid poly peptide [25] whereas the nucleation-elongation model has previously been linked to functional amyloids from E. coli and Pseudomonas [26].

In order to confirm the dominating mechanism of aggregation of the four peptides based on chemical kinetics, seeded aggregation in a regime of very low seed concentrations to monomeric concentration was conducted. This type of experiments delivers a direct means of probing the ability of fibrils to self-replicate $[24,27]$. The presence of preformed fibril seeds can accelerate the aggregation process by two different mechanisms, namely elongation and surface catalyzed secondary nucleation. The presence of low amounts of seeds eliminates the rate limiting step of primary nucleation when secondary nucleation is present but no changes in the kinetics will be observed when only primary nucleation and elongation is present as the low amounts of seed do not eliminate the need for more nuclei to be formed before the elongation process dominates. Indeed a decrease in the lag phase was observed with increasing seed concentration (up to $0.1 \%$ of the total protein mass present at the start of reaction) for PSM $\alpha 1$, PSM $\alpha 3$ and PSM $\beta 1$, Fig $1 \mathrm{~d}-\mathrm{f}$ and $1 \mathrm{~h}$ supporting the observation that secondary nucleation is the dominating molecular mechanism for the formation of new aggregates of PSM $\alpha 1, \operatorname{PSM} \alpha 3$ and PSM $\beta 1$ peptide [28]. Despite the very fast kinetics of PSM $\alpha 3$, the reduction in lag phase was significant and can be clearly observed, Fig. 1e. The aggregation kinetics of PSM $\beta 2$ was not affected by the presence of low amounts of seeds confirming the lack of self-replication processes in the form of surface catalyzed secondary nucleation, Fig. 1h. The general mechanism 
underlying formation of new aggregates from monomers of the PSM peptides from both primary and secondary pathways is shown in Fig. 1i.

\section{Elongation rates differ by a factor of $\mathbf{1 0 0 0}$ between fastest and slowest PSM}

The relative contributions of elongation rate constant $\left(k_{+}\right)$were investigated in presence of high concentration of preformed fibril seeds. The global fitting of the kinetic data yields a product of the elongation rate constant and the primary nucleation rate constant $\left(k_{n} k_{+}\right)$, however, in the presence of high amounts of preformed seeds, the intrinsic nucleation process becomes negligible, and hence the aggregation under this type of experiments is only dependent on elongation of the aggregates [28]. The initial increase in aggregate mass was measured through linear fits to the early points of the aggregation process, Fig. S3a, S3c, S3e and S3g [29, 30]. The estimated elongation rate constant for PSM $\alpha 1$ and PSM $\beta 2$ were found to be $0.2 \mathrm{mM} / \mathrm{h}^{2}$ and $0.5 \mathrm{mM} / \mathrm{h}^{2}$ respectively, differing by a factor of 2, which is insignificant. Contrary to PSM $\alpha 1$ and PSM $\beta 2$ the estimated elongation rate constant for PSM $\beta 1$, which aggregates at very low monomeric concentrations, was found to be $0.2 \mu \mathrm{M} / \mathrm{h}^{2}$ and hence a factor 1000 smaller than for PSM $\alpha 1$ and PSM $\beta 2$. In contrast, the elongation rate constant of PSM $\alpha 3$ was found to be $16.6 \mathrm{mM} / \mathrm{h}^{2}$ exceeding the values of PSM $\alpha 1$ and PSM $\beta 2 \sim 80$ and $\sim 35$ fold, respectively. The stronger effect of elongation of PSM $\alpha 3$ in comparison with other three peptides suggests that interactions with the fibrils increases the importance of PSM $\alpha 3$ fibrils in the assembly reactions compared with the assembly of free monomers of other peptides in the solution.

\section{Secondary structure analysis confirm $\alpha$-helical structure of PSM $\alpha 3$ and $\beta$-sheet structure for other PSMs}

The changes in secondary structure of the peptides following aggregation was monitored using synchrotron radiation circular dichroism (SRCD) spectroscopy and Fourier transform infrared (FTIR) spectroscopy. The CD spectra of monomers of all PSMs peptides prior to aggregation all show 
double minima at 208 and $222 \mathrm{~nm}$ indicative of $\alpha$-helical structure, Fig S4a. Upon aggregation the CD spectrum of the peptides changes displaying a single minimum at approximately $218 \mathrm{~nm}$ for PSM $\alpha 1$ and PSM $\alpha 4$, and at $220 \mathrm{~nm}$ for PSM $\beta 1$ and PSM $\beta 2$ indicative of $\beta$-sheet rich structure, Fig 2a. Despite the lack of sigmoidal aggregation curves for PSM $\alpha 4$ changes in the CD spectrum upon incubation was observed. This indicates a transition from $\alpha$-helical structure to a structure with increased $\beta$-sheet content upon aggregation and is consistent with data previously published $[4,5]$. The CD spectrum of aggregated PSM $\alpha 3$ is still displaying a double minimum with minima shifted to $208 \mathrm{~nm}$ and $228 \mathrm{~nm}$ indicative of $\alpha$-helical structure being present in the aggregates although this helical structure is different form that observed in the monomeric peptide. This observation is consistent with the reported cross- $\alpha$-helical structure of PSM $\alpha 3$ aggregates [13]. To further probe the contribution of the individual structural components to the $\mathrm{CD}$ spectra each spectrum was deconvoluted using the analysis programs Selecon3, Contin and CDSSTR in the DichroWeb server [31, 32], Fig. 2b and Table S1. Indeed the major structural contribution to the SRCD spectrum for PSM $\alpha 3$ aggregates is $\alpha$-helical ( 70\%). For PSM $\alpha 1$ and PSM $\alpha 4$ the major structural components are $\beta$-sheet (33\% and 35\% respectively) and unordered structure (39\% and $34 \%$ respectively). Despite the single minima indicative of predominantly $\beta$-sheet structure observed for PSM $\beta 1$ and PSM $\beta 2$ the major structural components are $\alpha$-helix (35\% and $40 \%$ respectively) and unordered structure (33\% and $35 \%$ respectively) with less contribution from $\beta$-sheet structure ( $24 \%$ and16\% respectively). No structural changes were observed for PSM $\alpha 2$ and $\delta$-toxin which upon incubation still displayed spectra characteristic of $\alpha$-helix, Fig. S4b. This is consistent with the lack of aggregation seen for these peptides by ThT fluorescence.

Consistent with the CD data, the FTIR spectra of PSM $\alpha 1$, PSM $\alpha 4$, PSM $\beta 1$ and PSM $\beta 2$ were found to be very similar with a well-defined intense peak at $\sim 1625 \mathrm{~cm}^{-1}$ indicative of amyloid $\beta$-sheet and a minor shoulder at $\sim 1667 \mathrm{~cm}^{-1}$ indicative of $\beta$-turns [33-35], Fig. 2c. The secondary structure 
composition of the fibrils was estimated using deconvolution of the spectra followed by conventional fitting program and summarized in Table S2, Fig 3b and FigS5a-e. In good agreement with the CD data and previous reports the FTIR spectra of PSM $\alpha 3$ aggregates shows significantly higher content of $\alpha$-helical structure relative to other PSMs peptide fibrils as shown by a more intense band in the spectrum of at $1655 \mathrm{~cm}^{-1}$, indicative of $\alpha$-helical structure [36].

Freshly formed PSMa3 aggregates are unstable but becomes stable through lateral association during maturation

The stability of the PSM aggregates was tested using CD spectroscopy. Fig 2e shows the CD signal at $220 \mathrm{~nm}$ for fibrils (PSM $\alpha 1, \mathrm{PSM} \alpha 3, \mathrm{PSM} \alpha 4$ and PSM $\beta 2$ ) from $25^{\circ} \mathrm{C}$ to $95^{\circ} \mathrm{C}$. All peptide fibrils spectra except PSM $\alpha 3$ indicate thermally stable $\beta$-sheet structure. Even at $95^{\circ} \mathrm{C}$, there is no indication of the loss of $\beta$-sheet structure of PSM $\alpha 1$, PSM $\alpha 4$ and PSM $\beta 2$, as judged by the stable negative peak at $220 \mathrm{~nm}$. However, freshly formed (1 h old) $\alpha$ PSM3 fibrils are thermally unstable as a loss of structure is seen above $50^{\circ} \mathrm{C}$ which is consistent with previous studies of fragments of PSM $\alpha 3$ [37]. Interestingly the lateral association of aggregates of PSM $\alpha 3$ upon further incubation (7 days, see Fig. 3) renders the fibrils thermally stable and no changes in structure is seen upon heating to $95{ }^{\circ} \mathrm{C}$ indicating that the lateral association of the aggregates stabilizes the structure. The stability of the aggregates towards chemical denaturants was tested using urea, Fig. S4c-d. Again aggregates of PSM $\alpha 3$ are the only ones susceptible towards disassembly (5M-8M urea) while no apparent effect is observed for PSM $\alpha 1$, PSM $\alpha 4$, PSM $\beta 1$ and PSM $\beta 2$ fibrils.

The morphological features of aggregates were examined using transmission electron microscopy (TEM). After seven days of incubation, PSM $\alpha 1$ formed stretches of entangled fibrils, Fig. 3a. In addition, bulky dense aggregate surrounded by a network of fibrils were observed. In contrast, PSM $\alpha 3$ incubated for $1 \mathrm{~h}$ generated short and unbranched fibrils, Fig.3b, which, upon two-day 
incubation, associates laterally to form stacks, Fig. S6c, and further associate to form entangles networks of fibrils after seven days of incubation, Fig. 3c. Aggregates of PSM $\beta 2$ showed entangled fibrils marginally thicker and more dispersed than PSM $\alpha 1$ and PSM $\alpha 3$, Fig. 3f. Aggregates of PSM $\beta 1$ also formed entangled networks of fibrils, Fig. 3e. No aggregated species could be observed for PSM $\alpha 2$ and $\delta$-toxin, Fig. S6a-b, consistent with the lack of aggregation as seen by the lack of increase in ThT fluorescence upon incubation. Interestingly, PSM $\alpha 4$ which lacked reproducible ThT kinetics but displayed $\beta$-sheet structure using CD and FTIR spectroscopy display very thin fibrils visible at higher magnification with some distribution of spherical aggregates, Fig. 3d. Overall, these data are in good agreement with the recorded kinetics and structural data.

\section{PSMa1 display promiscuous cross-seeding while other PSMs display selective cross-seeding}

\section{abilities}

The interplay between individual PSM peptides during formation of functional amyloids was investigated using cross-seeding experiments where the ability of aggregates of one PSM peptide to seed the aggregation of the other PSM peptides was tested. Cross-seeding experiments using 20\% preformed fibril seeds of PSM $\alpha 1$ and monomers of the rest of the PSM peptides were performed. It can be seen that the aggregation of all the other PSM peptides (PSM $\alpha 2-4$, PSM $\beta 1-2$, and $\delta$-toxin) is indeed accelerated by the presence of preformed fibrils seeds of PSM $\alpha 1$, Fig.4a, indicating that PSM $\alpha 1$ seeds not only effectively promoted self-aggregation through both elongation and secondary nucleation but also promoted cross-seeding between all PSMs peptides. Unlike PSM $\alpha 1$ the fast aggregating PSM $\alpha 3$ seeds are only capable of promoting self-aggregation and accelerating PSM 1 aggregation, Fig.4b and Fig. S7. The lag phase of PSM $\alpha 2$, PSM $\alpha 4, \operatorname{PSM} \beta 1 \& \delta$-toxin did not change upon addition of preformed PSM $\alpha 3$ seeds indicating that PSM $\alpha 1$ is more promiscuous in interaction 
with the other PSM peptides during aggregation. Interestingly the lag phase of PSM $\beta 2$ enhanced dramatically in presence of PSM $\alpha 3$ seeds, Fig.4b.

Like PSM $\alpha 3$ PSM $\beta 1$ is only capable of accelerating the aggregation of PSM $\alpha 1$, Fig 4c. Further, the effect of cross seeding other PSM peptides with PSM $\beta 2$ seeds was investigated. Pre-formed seeds of PSM $\beta 2$ were able to accelerate the aggregation of PSM $\alpha 1$ and PSM $\beta 1$ while also inducing aggregation of PSM $\alpha 2$ and $\delta$-toxin, Fig. $4 \mathrm{~d}$. None of the other PSM peptides were able to induce aggregation of these two PSM peptides which also do not aggregate on their own under conditions tested here. Furthermore, seeds of PSM $\beta 2$ caused insignificant changes in the fibrillations kinetics of PSM $\alpha 3$ and PSM $\alpha 4$.

Based on the cross-seeding analysis it is clear that the PSM peptides display selectivity in the interaction with preformed aggregates which cannot be explained simply by the sequence similarities with in the PSM $\alpha$ - or $\beta$-group indicating an intricate interplay between the various PSM peptides during biofilm formation. It is also clear that PSM $\alpha 1$ is the most promiscuous of the PSM peptides as aggregation of PSM $\alpha 1$ is accelerated by all the other PSM peptides that aggregate while also being able to accelerate the kinetics of aggregation of all the other PSM peptide even the ones that do not aggregate on their own. We therefor suggest a model describing the delicate interplay between individual PSM peptides in the formation of biofilm where the fast aggregating PSM $\alpha 3$ initiates the aggregation by forming unstable fibrils. These fibrils can then accelerate the aggregation of PSM 1 which forms stable fibrils capable of accelerating the aggregation of the majority of the remaining PSM peptides (PSM $\alpha 2$, PSM $\alpha 3$, PSM $\beta 1, \operatorname{PSM} \beta 2$ and $\delta$-toxin), Fig. 4e. In this way the fast kinetics of PSM 23 may act as a catalyst for the whole system of aggregation of PSMs peptides during biofilm formation.

\section{Discussion}


PSMs peptides are major determinants and play an important and diverse role in the biofilm matrix in S. aureus [11]. Previous studies have shown that PSMs from S. aureus form functional amyloids that contribute to biofilm integrity and provide resistance to disruption, which is critical to the virulence of medical device-associated infections $[6,38]$. However, there have been no efforts to date to establish a general picture for the self-assembly of PSMs peptides that brings together all the species in the aggregation cascade. In the present study, we have conducted a combination of detailed kinetic analysis with structural and morphological analysis to gain insight into the molecular and mechanistic steps, to determine how functional amyloid of PSMs, the biofilm determinant of Staphylococcus aureus, form and grow. This study involves studying separately the different process involved in the aggregation reaction i.e., initial nucleation steps, growth of fibrils and their amplification.

Earlier computational analysis of PSMs sequences (smallest staphylococcus toxins) already suggested that the peptides of individual families might display differential self-assembly properties [38]. We first determined the rates of the various microscopic steps (concentration dependent) associated with the aggregation of PSMs peptides. Our results have shown that under quiescent conditions, a dominant contribution to the formation of new aggregates is a fibril catalyzed secondary nucleation pathway, that is shared by both the variant of the $\alpha$-PSMs family (PSM $\alpha 1$ and PSM $\alpha 3$ ) which sustain the integrity of biofilms [6] and the $\beta$-PSM family (PSM $\beta 1$ ). In contrast to this, nucleation and elongation is the only processes contributing to the aggregation of PSM $\beta 2$, since the influence of secondary process that give rise to self-replication of aggregates is negligible for this peptide. It is remarkable that even though PSM $\alpha 1$ and PSM $\alpha 3$ possess seven identical and additional 10 similar amino acids in their sequence [10], they show distinct aggregation behavior as PSM $\alpha 3$ aggregates approximately 50 times faster than PSM $\alpha 1$, which specifies that the existence of distinct residue in PSM $\alpha 3$ might play a significant role in lowering the energy barrier for the steps in the conversion process of monomers to fibrils as observed for $A \beta$ peptides [18]. Furthermore the fibrils 
formed by PSM $\alpha 3$ was found to be initially unstable as also observed before [13] but upon further incubation the fibrils associate laterally to form more mature stable fibrils while fibrils of PSM 1 was stable from without the need for lateral association.

In the current study at quiescent conditions no aggregation kinetics were observed for PSM $\alpha 4$ despite observing $\beta$-sheet structure using CD and FTIR and monitoring very thin fibrils with TEM. Previous reports on the aggregation of PSMa4 involved incubation of up to 28 days or incubation under shaking conditions [37, 38]. As shaking conditions during aggregation is known to increase fragmentation due to shear forces [17] shaking conditions were excluded in the present study. Compared to the other PSM peptides PSMa4 is the one with both the lowest solubility score and the lowest calculated aggregation propensity when computing these using the CamSol algorithm [39, 40], Fig S8a-b and Table S3. This could possibly explain the need for long incubation time and shaking conditions during aggregation.

Functional amyloids from gram-negative bacteria are mainly composed of a single protein such as CsgA in E. coli curli and FapC in Pseudomonas [41, 42]. Along with the proteins incorporated into the functional amyloids a whole range of auxiliary proteins is expressed simultaneously. In the grampositive bacteria $S$. aureus the functional amyloids in biofilms is made up of the different PSM peptides [6]. The model suggested here account for the role of individual PSM peptides during formation of functional amyloids to stabilize the biofilm hence allowing the bacteria an efficient way to from functional amyloids in a very short amount of time but at the cost of stability. The stability is later gained by the aggregation of other PSM. The most proinflammatory and cytotoxic PSM $\alpha 3$ [12], boosts the reaction of PSM $\alpha 1$ kinetics followed by enhancement of aggregation kinetics of rest of the PSMs peptides, which likely play a key role in stabilizing the biofilm matrix [6] and influences the biofilm development and structuring activities [14]. The highly stable amyloidal structures thus serve 
as the building blocks cementing the biofilm and creating the rigidity that can explain the resistance of amyloid-containing biofilms. Overall, we note that the rates of individual kinetic steps in the process can differ by several orders of magnitude between different variants, whereas in previous reports a vast structural diversity of amyloid like structures have been also reported for PSMs peptides [37]. Further, in vitro studies also confirmed that contrary to what previously thought [6], not all PSMs forms amyloid structures even at higher concentrations.

We conclude that the outcomes presented in this article may have significant implications for understanding the aggregation process of PSMs peptides during biofilm formation. These findings indicate a molecular interplay between individual PSM peptides during accumulation of PSMs amyloid fibrils in biofilms. This also suggest an important approach for suppressing the biofilm growth of S. aureus as PSMs have critical role during infection and represent a promising target for anti-staphylococcal activity [43]. Recently potential inhibitors of A $\beta$ aggregation in Alzheimer's and a-synuclein in Parkinson's disease have been found to inhibit self-replication by secondary nucleation being the most promising candidate [44]. In the case of $S$. aureus biofilm forming amyloids this could also be a potential strategy as several of the PSM peptides aggregated through a secondary nucleation dominated mechanism. This could be possible by using inhibitors of amyloid formation as numerous studies have demonstrated that inhibitors of aggregation also tend to inhibit biofilm formation $[45,46]$. In the context of the development of biofilm formation, the key processes and mechanisms revealed in this study is likely to contribute to the difficulty in controlling and to understanding the role of amyloid growth as a potentially limiting factor of biofilm formation.

\section{Material and methods:}

Peptides and reagents: N-terminally formylated PSM peptides (> 95\% purity) were purchased from GenScript Biotech, Netherlands. Thioflavin $\mathrm{T}$ (ThT), trifluoroacetic acid (TFA) and 
hexafluoroisopropanol (HFIP) were purchased from Sigma Aldrich. Dimethyl-sulfoxide (DMSO) was purchased from Merck. Ultra-pure water was used for the entire study.

Peptide pre-treatment: Lyophilized PSMs peptide stocks were dissolved to a concentration of 0.5 $\mathrm{mgml}^{-1}$ in a 1:1 mixture of HFIP and TFA followed by a $5 \times 20$ seconds sonication with 30 seconds intervals using a probe sonicator, and incubation at room temperature for one hour. The HFIP/TFA mixture was evaporated by speedvac at $1000 \mathrm{rpm}$ for 3 hour at room temperature. Dried peptide stocks were stored at $-80{ }^{\circ} \mathrm{C}$ prior to use.

Preparation of samples for kinetics experiments: All kinetic experiments were performed in 96well black Corning polystyrene half-area microtiter plates with a non-binding surface incubated at 37 ${ }^{\circ} \mathrm{C}$ in a Fluostar Omega plate reader (BMG Labtech, Germany). Aliquots of purified PSMs were thawed and dissolved in dimethyl sulfoxide (DMSO) to a concentration of $10 \mathrm{mgml}^{-1}$ prior to use. Freshly dissolved peptides were diluted into sterile $\mathrm{ddH}_{2} \mathrm{O}$ containing $0.04 \mathrm{mM}$ ThT. $100 \mu \mathrm{L}$ of samples were added to each well and the plate was sealed to prevent evaporation. The ThT fluorescence was measured every 10 minutes with an excitation filter of $450 \mathrm{~nm}$ and an emission filter of $482 \mathrm{~nm}$ at quiescent conditions. However, for PSM $\alpha 3$ the measurement was done every $15 \mathrm{~s}$ with same excitation and emission wavelength. The ThT fluorescence was followed by three repeats of each monomer concentration.

Pre-seeded kinetic assay: Fibrils of different peptides were collected and sonicated for 2 minutes in a sonicator bath at room temperature in low bind Eppendorf tubes (Axygen). Seeds were added to fresh monomer of corresponding peptide immediately before ThT measurements. In cross-seeding experiment, seeds (PSM $\alpha 1$ and $\alpha 3$, PSM $\beta 2$ ) were added to monomer of all other PSMs variants. ThT fluorescence was observed in the plate reader every 10 minutes under quiescent conditions. 
Calculation of the elongation rate constant: To estimate the rates of fibril elongation seeded aggregation with high concentration of preformed fibril seeds (20-50\% of monomeric equivalents concentration) and fixed monomeric concentrations $(0.25 \mathrm{mg} / \mathrm{mL}$ of PSM $\alpha 1,0.5 \mathrm{mg} / \mathrm{mL}$ of PSM $\alpha 3$, $0.025 \mathrm{mg} / \mathrm{mL}$ for PSM $\beta 1$, and $0.25 \mathrm{mg} / \mathrm{mL}$ for PSM $\beta 2$ ) was performed. The initial gradients (first 120 min for PSM $\alpha 1$, PSM $\beta 1$ and PSM $\beta 2$ and the first 120 seconds for PSM $\alpha 3$ ) of the kinetic curves were determined and plotted against the monomer concentration. Data points at higher concentration were excluded due to saturation effect of elongation.

Far-UV circular dichroism (CD) spectroscopy: CD was performed on a JASCO-810 Spectrophotometer at $25{ }^{\circ} \mathrm{C}$, wavelength 200 to $250 \mathrm{~nm}$ with a step size of $0.5 \mathrm{~nm}, 2 \mathrm{~nm}$ bandwidth and a scan speed of $50 \mathrm{~nm} / \mathrm{min}$. Samples were loaded in a 1-mm Quartz cuvette. Triplicate samples containing various peptide concentrations of each freshly dissolved peptides were pelleted and supernatant was transferred to clean sterile tube. The remaining pellet was resuspended in the same volume of $\mathrm{ddH}_{2} \mathrm{O}$ followed by bath sonication. For each sample, the average of five scans were recorded and corrected for baseline contribution and the $\mathrm{ddH}_{2} \mathrm{O}$ signal was subtracted.

Synchotron radiation circular dichroism spectroscopy (SRCD): The SRCD spectra of the various PSM fibrils were collected at the AU-CD beamline of the ASTRID2 synchrotron, Aarhus University, Denmark. Three to five successive scans over the wavelength range from 170 to $280 \mathrm{~nm}$ were recorded at $25^{\circ} \mathrm{C}$, using a $0.1 \mathrm{~mm}$ path length cuvette, at $1 \mathrm{~nm}$ intervals with a dwell time of 2 sec. All SRCD spectra were processed and subtracted from their respective averaged baseline (solution containing all components of the sample, except the protein), smoothing with a 7 pt Savitzky-Golay filter, and expressing the final SRCD spectra in mean residual ellipticity. The SRCD spectra of the individual PSM fibrils samples were deconvoluted using DichroWeb [31, 32] to obtain the contribution from individual structural components. Each spectrum was fitted using the analysis 
programs Selecon3, Contin and CDSSTR with the SP175 reference data set [47] and an average of the structural component contributions from the three analysis programs was used.

Fourier transform Infrared Spectroscopy (FTIR): Tensor 27 FTIR spectrometer (Bruker) equipped with attenuated total reflection accessory with a continuous flow of $\mathrm{N}_{2}$ gas was used to collect spectra of different aliquots. All samples were dried with $\mathrm{N}_{2}$ gas. For each spectrum 64 interferograms were accumulated with a spectral resolution of $2 \mathrm{~cm}^{-1}$ in the range from 1000 to 3998 $\mathrm{cm}^{-1}$. The data were processed by baseline correction and interfering signals from $\mathrm{H}_{2} \mathrm{O}$ and $\mathrm{CO}_{2}$ were removed using the atmospheric compensation filter. Further, peak positions were assigned where the second order derivative had local minima and the intensity was modeled by Gaussian curve fitting using the OPUS 5.5 software. All absorbance spectra were normalized for comparative study.

Transmission electron microscopy (TEM): Fibrillated samples $(5 \mu \mathrm{L})$ of all peptides at various concentrations were placed on carbon coated formvar grid (EM resolutions), incubated for 2 min, washed with $\mathrm{ddH}_{2} \mathrm{O}$ followed by negative staining with $2 \%$ uranyl acetate for 2 min. Further, the grids were washed twice with $\mathrm{ddH}_{2} \mathrm{O}$ and blotted dry on filter paper. The samples were examined using a Morgagni 268 from FEI Phillips Electron microscopy, equipped with a CCD digital camera, and operated at an accelerating voltage of $80 \mathrm{KV}$.

Fibril stability: CD spectra of fibrils (PSM $\alpha 1, \alpha 3, \alpha 4, \operatorname{PSM} \beta 1$ and $\beta 2$ ) were recorded from 25 to 95 ${ }^{\circ} \mathrm{C}$ with a step size of $0.1^{\circ} \mathrm{C}$ at $220 \mathrm{~nm}$. Stability towards denaturants was tested by dialyzing fibrils (PSM $\alpha 1, \alpha 3, \alpha 4, \operatorname{PSM} \beta 1$ and $\beta 2)$ containing various concentration of urea $(0-8 \mathrm{M})$ for $24 \mathrm{~h}$.

Conflict of interest: The authors declare no conflict of interest.

\section{Acknowledgements:}


This work was supported by Aarhus University Research Foundation. MA is the recipient of a Starting Grant from Aarhus University Research Foundation. Furthermore we acknowledge the award of beam time on the AU-CD beam line at ASTRID2, under project number ISA-20-1013

\section{Author contribution:}

M.Z. and M.A. designed experiments, M.Z. carried out the experiments, M.Z. and M.A. analyzed the data, M.Z. and M.A. wrote the manuscript, M.A. secured the funding.

\section{References:}

[1] C.L. Pham, A.H. Kwan, M. Sunde, Functional amyloid: widespread in Nature, diverse in purpose, Essays in biochemistry, 56 (2014) 207-219.

[2] S.K. Maji, M.H. Perrin, M.R. Sawaya, S. Jessberger, K. Vadodaria, R.A. Rissman, P.S. Singru, K.P. Nilsson, R. Simon, D. Schubert, D. Eisenberg, J. Rivier, P. Sawchenko, W. Vale, R. Riek, Functional amyloids as natural storage of peptide hormones in pituitary secretory granules, Science (New York, N.Y.), 325 (2009) 328-332.

[3] M.L. Evans, M.R. Chapman, Curli biogenesis: order out of disorder, Biochimica et biophysica acta, 1843 (2014) 1551-1558.

[4] M.S. Dueholm, S.V. Petersen, M. Sonderkaer, P. Larsen, G. Christiansen, K.L. Hein, J.J. Enghild, J.L. Nielsen, K.L. Nielsen, P.H. Nielsen, D.E. Otzen, Functional amyloid in Pseudomonas, Molecular microbiology, 77 (2010) 1009-1020.

[5] D. Romero, C. Aguilar, R. Losick, R. Kolter, Amyloid fibers provide structural integrity to Bacillus subtilis biofilms, Proc Natl Acad Sci U S A, 107 (2010) 2230-2234.

[6] K. Schwartz, A.K. Syed, R.E. Stephenson, A.H. Rickard, B.R. Boles, Functional amyloids composed of phenol soluble modulins stabilize Staphylococcus aureus biofilms, PLoS pathogens, 8 (2012) e1002744.

[7] N. Van Gerven, S.E. Van der Verren, D.M. Reiter, H. Remaut, The Role of Functional Amyloids in Bacterial Virulence, Journal of molecular biology, 430 (2018) 3657-3684.

[8] P.M. Gallo, G.J. Rapsinski, R.P. Wilson, G.O. Oppong, U. Sriram, M. Goulian, B. Buttaro, R.

Caricchio, S. Gallucci, C. Tukel, Amyloid-DNA Composites of Bacterial Biofilms Stimulate Autoimmunity, Immunity, 42 (2015) 1171-1184.

[9] L.S. Marmont, J.D. Rich, J.C. Whitney, G.B. Whitfield, H. Almblad, H. Robinson, M.R. Parsek, J.J. Harrison, Oligomeric lipoprotein PelC guides Pel polysaccharide export across the outer membrane of Pseudomonas aeruginosa, Proceedings of the National Academy of Sciences of the United States of America, 114 (2017) 2892-2897.

[10] A. Bleem, R. Francisco, J.D. Bryers, V. Daggett, Designed alpha-sheet peptides suppress amyloid formation in Staphylococcus aureus biofilms, NPJ biofilms and microbiomes, 3 (2017) 16. [11] A. Peschel, M. Otto, Phenol-soluble modulins and staphylococcal infection, Nature reviews. Microbiology, 11 (2013) 667-673.

[12] R. Wang, K.R. Braughton, D. Kretschmer, T.H. Bach, S.Y. Queck, M. Li, A.D. Kennedy, D.W. Dorward, S.J. Klebanoff, A. Peschel, F.R. DeLeo, M. Otto, Identification of novel cytolytic peptides as key virulence determinants for community-associated MRSA, Nat Med, 13 (2007) 1510-1514. 
[13] E. Tayeb-Fligelman, O. Tabachnikov, A. Moshe, O. Goldshmidt-Tran, M.R. Sawaya, N. Coquelle, J.P. Colletier, M. Landau, The cytotoxic Staphylococcus aureus PSMalpha3 reveals a cross-alpha amyloid-like fibril, Science, 355 (2017) 831-833.

[14] S. Periasamy, H.S. Joo, A.C. Duong, T.H. Bach, V.Y. Tan, S.S. Chatterjee, G.Y. Cheung, M. Otto, How Staphylococcus aureus biofilms develop their characteristic structure, Proceedings of the National Academy of Sciences of the United States of America, 109 (2012) 1281-1286.

[15] T.P. Knowles, C.A. Waudby, G.L. Devlin, S.I. Cohen, A. Aguzzi, M. Vendruscolo, E.M. Terentjev, M.E. Welland, C.M. Dobson, An analytical solution to the kinetics of breakable filament assembly, Science, 326 (2009) 1533-1537.

[16] G. Meisl, J.B. Kirkegaard, P. Arosio, T.C. Michaels, M. Vendruscolo, C.M. Dobson, S. Linse, Molecular mechanisms of protein aggregation from global fitting of kinetic models, Nature protocol, 11 (2016) 252-272.

[17] S.I. Cohen, S. Linse, L.M. Luheshi, E. Hellstrand, D.A. White, L. Rajah, D.E. Otzen, M. Vendruscolo, C.M. Dobson, T.P. Knowles, Proliferation of amyloid-beta42 aggregates occurs through a secondary nucleation mechanism, Proc Natl Acad Sci U S A, 110 (2013) 9758-9763. [18] G. Meisl, X. Yang, E. Hellstrand, B. Frohm, J.B. Kirkegaard, S.I. Cohen, C.M. Dobson, S. Linse, T.P. Knowles, Differences in nucleation behavior underlie the contrasting aggregation kinetics of the Abeta40 and Abeta42 peptides, Proc Natl Acad Sci U S A, 111 (2014) 9384-9389.

[19] S.R. Collins, A. Douglass, R.D. Vale, J.S. Weissman, Mechanism of prion propagation: amyloid growth occurs by monomer addition, PLoS Biol, 2 (2004) e321.

[20] H. LeVine, 3rd, Thioflavine T interaction with synthetic Alzheimer's disease beta-amyloid peptides: detection of amyloid aggregation in solution, Protein Sci, 2 (1993) 404-410.

[21] G. Meisl, J.B. Kirkegaard, P. Arosio, T.C. Michaels, M. Vendruscolo, C.M. Dobson, S. Linse,

T.P. Knowles, Molecular mechanisms of protein aggregation from global fitting of kinetic models, Nature protocols, 11 (2016) 252-272.

[22] V. Fodera, F. Librizzi, M. Groenning, M. van de Weert, M. Leone, Secondary nucleation and accessible surface in insulin amyloid fibril formation, The journal of physical chemistry. B, 112 (2008) 3853-3858.

[23] R. Gaspar, G. Meisl, A.K. Buell, L. Young, C.F. Kaminski, T.P.J. Knowles, E. Sparr, S. Linse, Secondary nucleation of monomers on fibril surface dominates alpha-synuclein aggregation and provides autocatalytic amyloid amplification, Quarterly reviews of biophysics, 50 (2017) e6.

[24] A.K. Buell, C. Galvagnion, R. Gaspar, E. Sparr, M. Vendruscolo, T.P. Knowles, S. Linse, C.M. Dobson, Solution conditions determine the relative importance of nucleation and growth processes in alpha-synuclein aggregation, Proc Natl Acad Sci U S A, 111 (2014) 7671-7676.

[25] A.M. Ruschak, A.D. Miranker, Fiber-dependent amyloid formation as catalysis of an existing reaction pathway, Proceedings of the National Academy of Sciences of the United States of America, 104 (2007) 12341-12346.

[26] M. Andreasen, G. Meisl, J.D. Taylor, T.C.T. Michaels, A. Levin, D.E. Otzen, M.R. Chapman, C.M. Dobson, S.J. Matthews, T.P.J. Knowles, Physical Determinants of Amyloid Assembly in Biofilm Formation, mBio, 10 (2019).

[27] P. Arosio, R. Cukalevski, B. Frohm, T.P. Knowles, S. Linse, Quantification of the concentration of Abeta42 propagons during the lag phase by an amyloid chain reaction assay, J Am Chem Soc, 136 (2014) 219-225.

[28] S.I. Cohen, M. Vendruscolo, C.M. Dobson, T.P. Knowles, From macroscopic measurements to microscopic mechanisms of protein aggregation, Journal of molecular biology, 421 (2012) 160-171. [29] T. Weiffert, G. Meisl, P. Flagmeier, S. De, C.J.R. Dunning, B. Frohm, H. Zetterberg, K. Blennow, E. Portelius, D. Klenerman, C.M. Dobson, T.P.J. Knowles, S. Linse, Increased Secondary 
Nucleation Underlies Accelerated Aggregation of the Four-Residue N-Terminally Truncated Abeta42 Species Abeta5-42, ACS chemical neuroscience, (2019).

[30] C.B. Rasmussen, G. Christiansen, B.S. Vad, C. Lynggaard, J.J. Enghild, M. Andreasen, D.

Otzen, Imperfect repeats in the functional amyloid protein FapC reduce the tendency to fragment during fibrillation, Protein Sci, 28 (2019) 633-642.

[31] L. Whitmore, B.A. Wallace, Protein secondary structure analyses from circular dichroism spectroscopy: methods and reference databases, Biopolymers, 89 (2008) 392-400.

[32] L. Whitmore, B.A. Wallace, DICHROWEB, an online server for protein secondary structure analyses from circular dichroism spectroscopic data, Nucleic Acids Res, 32 (2004) W668-673.

[33] M. Gasset, M.A. Baldwin, D.H. Lloyd, J.M. Gabriel, D.M. Holtzman, F. Cohen, R. Fletterick, S.B. Prusiner, Predicted alpha-helical regions of the prion protein when synthesized as peptides form amyloid, Proceedings of the National Academy of Sciences of the United States of America, 89 (1992) 10940-10944.

[34] M.S. Dueholm, S.B. Nielsen, K.L. Hein, P. Nissen, M. Chapman, G. Christiansen, P.H. Nielsen, D.E. Otzen, Fibrillation of the major curli subunit CsgA under a wide range of conditions implies a robust design of aggregation, Biochemistry, 50 (2011) 8281-8290.

[35] G. Zandomeneghi, M.R. Krebs, M.G. McCammon, M. Fandrich, FTIR reveals structural differences between native beta-sheet proteins and amyloid fibrils, Protein Sci, 13 (2004) 3314-3321.

[36] J. Kong, S. Yu, Fourier transform infrared spectroscopic analysis of protein secondary structures, Acta Biochim Biophys Sin (Shanghai), 39 (2007) 549-559.

[37] N. Salinas, J.P. Colletier, A. Moshe, M. Landau, Extreme amyloid polymorphism in Staphylococcus aureus virulent PSMalpha peptides, Nat Commun, 9 (2018) 3512.

[38] P. Marinelli, I. Pallares, S. Navarro, S. Ventura, Dissecting the contribution of Staphylococcus aureus alpha-phenol-soluble modulins to biofilm amyloid structure, Scientific reports, 6 (2016) 34552.

[39] P. Sormanni, F.A. Aprile, M. Vendruscolo, The CamSol method of rational design of protein mutants with enhanced solubility, J Mol Biol, 427 (2015) 478-490.

[40] P. Sormanni, L. Amery, S. Ekizoglou, M. Vendruscolo, B. Popovic, Rapid and accurate in silico solubility screening of a monoclonal antibody library, Scientific reports, 7 (2017) 8200.

[41] M.R. Chapman, L.S. Robinson, J.S. Pinkner, R. Roth, J. Heuser, M. Hammar, S. Normark, S.J. Hultgren, Role of Escherichia coli curli operons in directing amyloid fiber formation, Science, 295 (2002) 851-855.

[42] M.S. Dueholm, S.V. Petersen, M. Sonderkaer, P. Larsen, G. Christiansen, K.L. Hein, J.J. Enghild, J.L. Nielsen, K.L. Nielsen, P.H. Nielsen, D.E. Otzen, Functional amyloid in Pseudomonas, Mol Microbiol, (2010).

[43] G.Y. Cheung, H.S. Joo, S.S. Chatterjee, M. Otto, Phenol-soluble modulins--critical determinants of staphylococcal virulence, FEMS microbiology reviews, 38 (2014) 698-719.

[44] S.I.A. Cohen, P. Arosio, J. Presto, F.R. Kurudenkandy, H. Biverstal, L. Dolfe, C. Dunning, X. Yang, B. Frohm, M. Vendruscolo, J. Johansson, C.M. Dobson, A. Fisahn, T.P.J. Knowles, S. Linse, A molecular chaperone breaks the catalytic cycle that generates toxic Abeta oligomers, Nature structural \& molecular biology, 22 (2015) 207-213.

[45] K. Arita-Morioka, K. Yamanaka, Y. Mizunoe, T. Ogura, S. Sugimoto, Novel strategy for biofilm inhibition by using small molecules targeting molecular chaperone DnaK, Antimicrobial agents and chemotherapy, 59 (2015) 633-641.

[46] K.I. Arita-Morioka, K. Yamanaka, Y. Mizunoe, Y. Tanaka, T. Ogura, Inhibitory effects of Myricetin derivatives on curli-dependent biofilm formation in Escherichia coli, Scientific reports, 8 (2018) 8452. 
bioRxiv preprint doi: https://doi.org/10.1101/2020.04.01.020610; this version posted April 2, 2020. The copyright holder for this preprint (which was not certified by peer review) is the author/funder. All rights reserved. No reuse allowed without permission.

[47] J.G. Lees, A.J. Miles, F. Wien, B.A. Wallace, A reference database for circular dichroism spectroscopy covering fold and secondary structure space, Bioinformatics, 22 (2006) 1955-1962. 


\section{Figures}
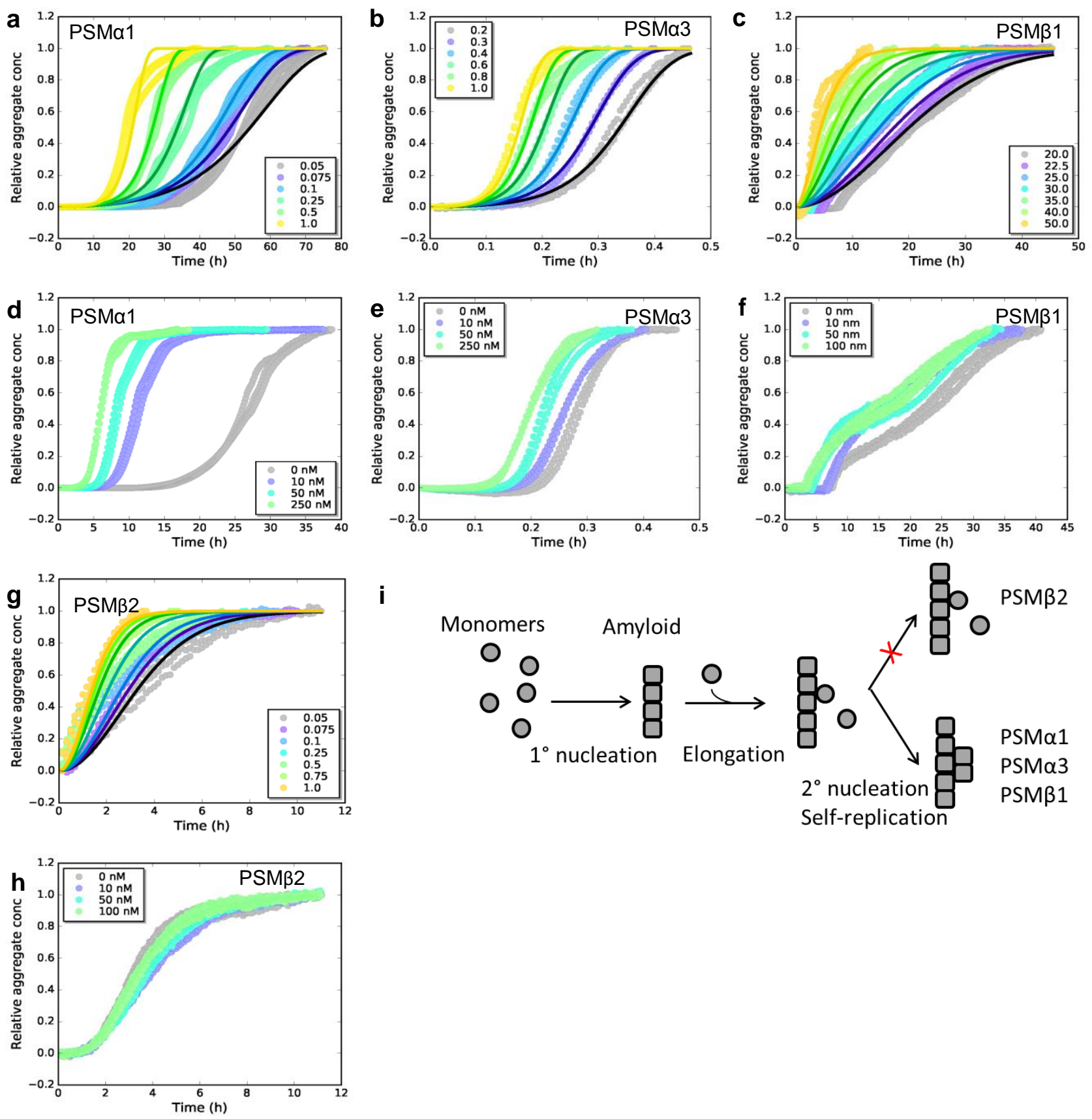

Fig. 1: Experimental ThT kinetic data for the aggregation of PSMs peptides from monomeric samples. (a) Aggregation of PSM $\alpha 1(0.05 \mathrm{mg} / \mathrm{mL}$ to $1.0 \mathrm{mg} / \mathrm{mL})$. The data are well fitted by a secondary nucleation model. (b) Aggregation of PSM $\alpha 3(0.2 \mathrm{mg} / \mathrm{mL}$ to $1.0 \mathrm{mg} / \mathrm{mL})$. The data are well fitted by a secondary nucleation model. (c) Aggregation of PSM $\beta 1$ (20 $\mu \mathrm{g} / \mathrm{mL}$ to $50 \mu \mathrm{g} / \mathrm{mL})$. The data are well fitted by a secondary nucleation model. (d) Aggregation of PSM $\alpha 1$ in the presence 
and absence of low concentrations of preformed seeds, at a monomer concentration of $0.5 \mathrm{mg} / \mathrm{mL}$ and seed concentration of 0 to $250 \mathrm{nM}$ monomer equivalents. Significant effects of added seeds on the rate of aggregation were observed. (e) Aggregation of PSM $\alpha 3$ in the presence and absence of low concentrations of preformed seeds, at a monomer concentration of $0.4 \mathrm{mg} / \mathrm{mL}$ and seed concentration of 0 to $250 \mathrm{nM}$ monomer equivalents. Significant effects of added seeds on the rate of aggregation were observed but lower than $\alpha$ PSM1. (f) Aggregation of PSM $\beta 1$ in the presence and absence of low concentration of preformed seeds, at a monomer concentration of $0.025 \mathrm{mg} / \mathrm{mL}$ and seed concentration of 0 to $100 \mathrm{nM}$ monomer equivalents. Significant effects of added seeds on the rate of aggregation were observed but lower than $\alpha$ PSM1. (g) Aggregation of PSM $\beta 2(0.05 \mathrm{mg} / \mathrm{mL}$ to 1.0 $\mathrm{mg} / \mathrm{mL}$ ). The data are well fitted by a nucleation-elongation model. (h) Aggregation of PSM $\beta 2$ in the presence and absence of low concentration of preformed seeds, at a monomer concentration of 0.5 $\mathrm{mg} / \mathrm{mL}$ and seed concentration of 0 to $100 \mathrm{nM}$ monomer equivalents. No significant effects of added seeds on the rate of aggregation are evident. (i) A schematic illustration of the microscopic steps involved in PSM aggregation. Monomers of PSM $\beta 2$ nucleate through primary nucleation with rate constant $\mathrm{k}_{\mathrm{n}}$ and the aggregates grow by addition of monomers to the fibril end with rate constant $\mathrm{k}_{+}$. In addition to primary nucleation and elongation monomers of PSM $\alpha 1, \mathrm{PSM} \alpha 3$ and PSM $\beta 1$ nucleate through secondary nucleation on the surface of an already existing aggregate with secondary rate constant $\mathrm{k}_{2}$. All kinetic experiments were carried out in triplicates. Parameters from the data fitting are summarized in Table 1. 

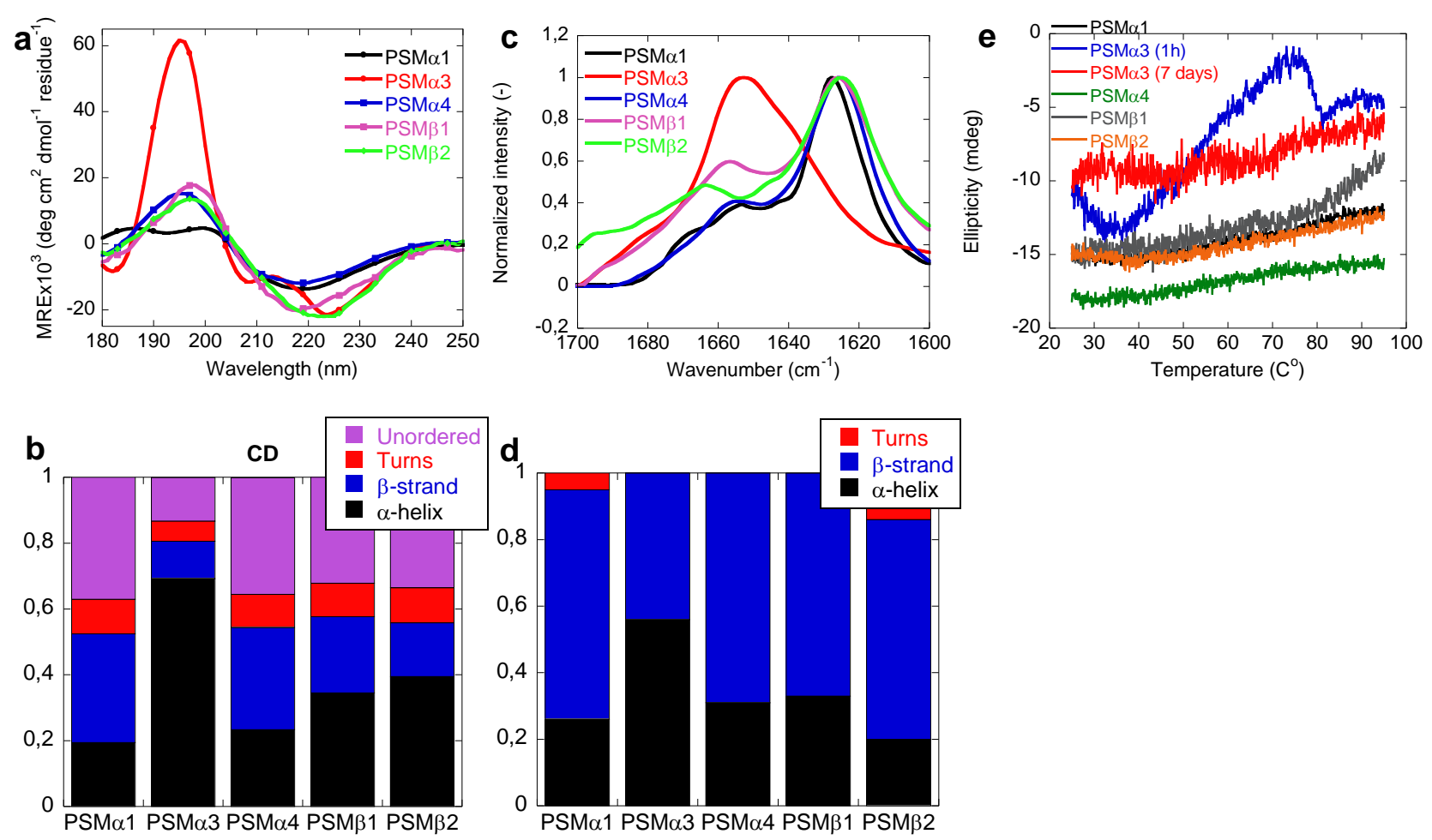

Figure 2: Structural comparison of fibrils formed by different PSMs variants. (a) Synchrotron radiation (SR) Far UV-CD spectra of PSM $\alpha 1$, PSM $\alpha 3$, PSM $\alpha 4$, PSM $\beta 1$ and PSM $\beta 2$ fibrils recorded after seven days of incubated samples except for PSM $\alpha 3$ which is recorded after one hour of incubated samples. (b) Deconvolution of the SR-CD spectra of fibrils of PSM variants into the individual structural components. (c) Attenuated total internal reflection Fourier transform infrared (ATR-FTIR) spectroscopy of the amide I' region $\left(1600-1700 \mathrm{~cm}^{-1}\right)$ of fibrils of PSMs variants. PSM $\alpha 1$, PSM $\alpha 4$, PSM $\beta 1$ and PSM $\beta 2$ show a peak at $1625 \mathrm{~cm}^{-1}$ corresponding to rigid amyloid fibrils. In contrast, PSM $\alpha 3$ shows main peaks at and $1654 \mathrm{~cm}^{-1}$, with the latter indicating on more disordered fibrils. (d) Deconvolution of the FTIR spectra of fibrils of the PSM variants into the individual structural components. (e) $\mathrm{CD}$ thermal scans from 20 to $95^{\circ} \mathrm{C}$ of PSM $\alpha 1, \operatorname{PSM} \alpha 3$ (1h), PSM $\alpha 4$, PSM $\beta 1$ and PSM $\beta 2$ fibrils. 


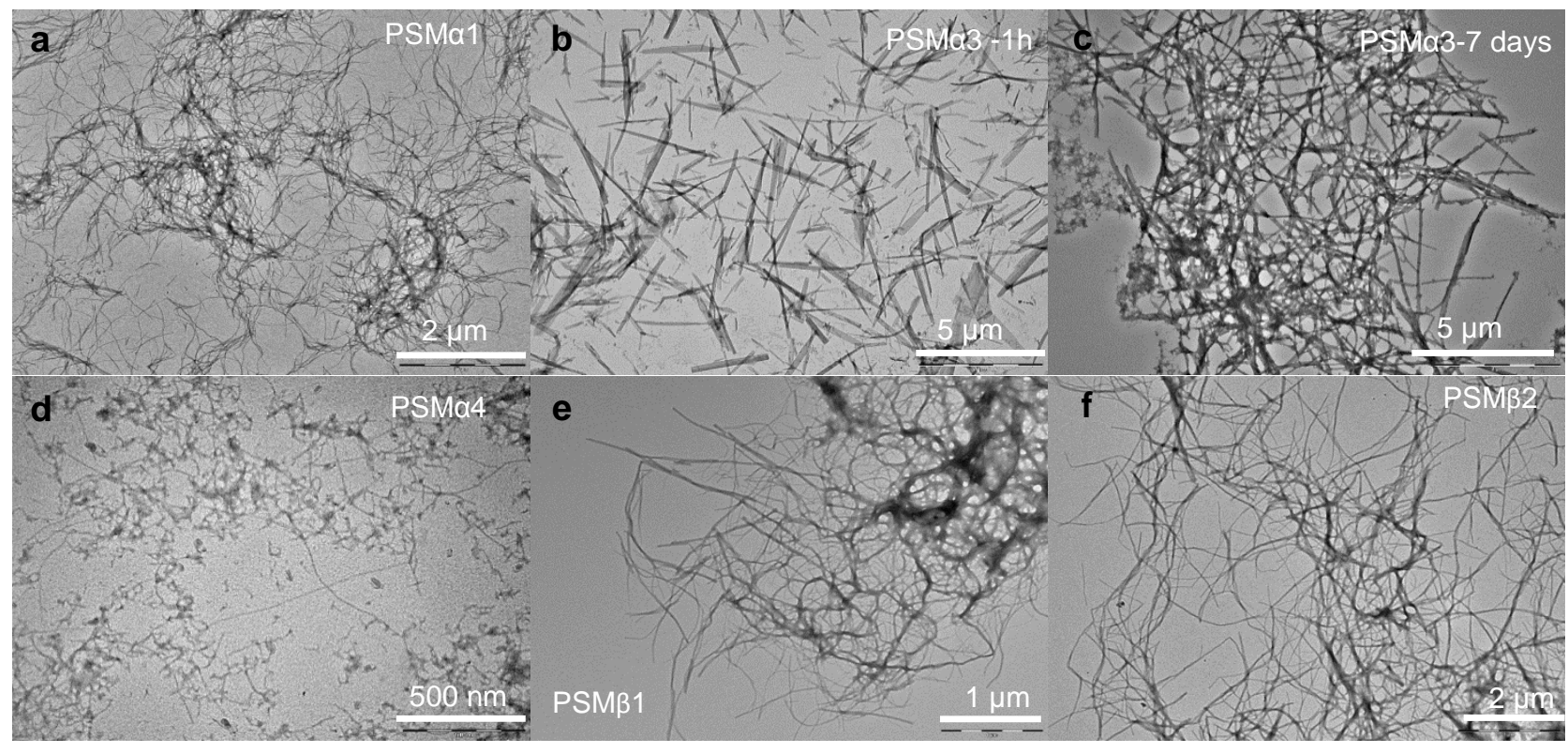

Figure 3: Morphology of aggregates of PSMs peptides. Transmission electron microscopic image of the end state of reaction for samples initially composed of (a) PSM $\alpha 1$ fibrils, (b) PSM $\alpha 3$ fibrils after 1h of incubation, (c) PSM $\alpha 3$ fibrils after 7 days of incubation, (d) PSM $\alpha 4$ fibrils, (e) PSM 1 fibrils and (f) PSM $\beta 2$ fibrils. Please note that scale bar changes. 

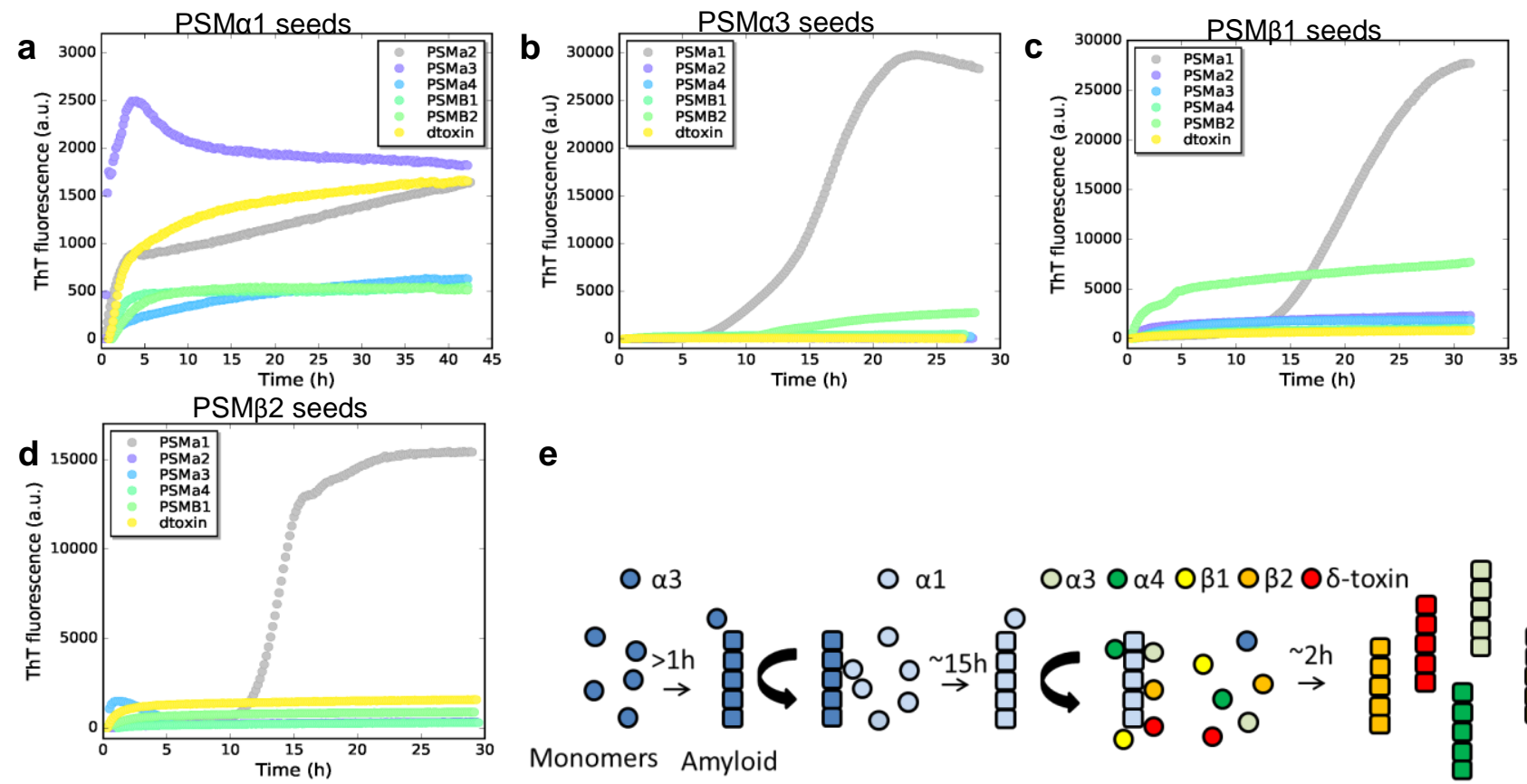

e

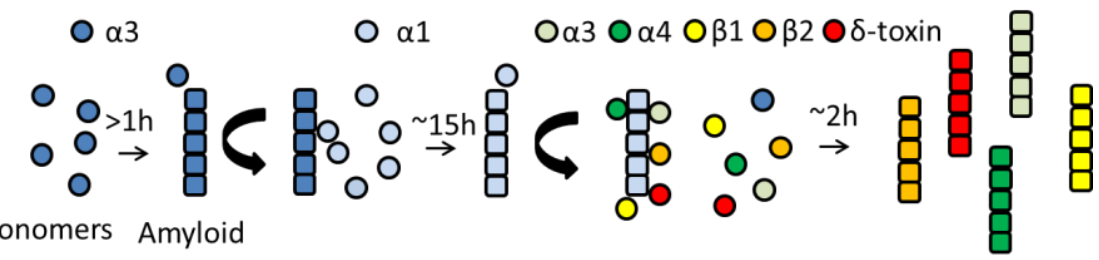

Figure 4: Cross seeding PSMs variant. (a) Aggregation of PSM $\alpha 2$, PSM $\alpha 3$, PSM $\alpha 4$, PSM $\beta 1$, PSM $\beta 2$ and $\delta$-toxin $(0.25 \mathrm{mg} / \mathrm{mL})$ in the presence of $20 \%$ preformed PSM $\alpha 1$ seeds. (b) Aggregation of PSM $\alpha 1, \mathrm{PSM} \alpha 2, \mathrm{PSM} \alpha 4, \mathrm{PSM} \beta 1, \mathrm{PSM} \beta 2$ and $\delta$-toxin $(0.25 \mathrm{mg} / \mathrm{mL})$ in the presence of $20 \%$ preformed PSM $\alpha 3$ seeds. (c) Aggregation of PSM $\alpha 1, \mathrm{PSM} \alpha 2, \mathrm{PSM} \alpha 3, \mathrm{PSM} \alpha 4, \mathrm{PSM} \beta 2$ and $\delta$-toxin $(0.25 \mathrm{mg} / \mathrm{mL})$ in the presence of $20 \%$ preformed PSM $\beta 1$ seeds. (d) Aggregation of PSM $\alpha 1$, PSM $\alpha 2$, PSM $\alpha 3$, PSM $\alpha 4$, PSM $\beta 1$ and $\delta$-toxin $(0.25 \mathrm{mg} / \mathrm{mL})$ in the presence of $20 \%$ preformed PSM $\beta 2$ seeds. (e) Schematic representation of the cross seeding interactions between the PSM variants during biofilm formation. 
Table 1: Kinetic parameters obtained from fitting of data in Figure 1 using the web server AmyloFit. $\mathrm{n}_{\mathrm{c}}$ and $\mathrm{n}_{2}$ are the reaction order of the primary and secondary nucleation process respectively, $\mathrm{k}_{\mathrm{n}}$ and $\mathrm{k}_{2}$ are rate constants for the primary and secondary nucleation process and $\mathrm{k}_{+}$is the rate constant for the elongation of existing fibrils.

\begin{tabular}{|l|l|l|l|l|}
\hline Parameters & PSM $\alpha 1$ & PSM $\alpha 3$ & PSM $\beta 1$ & PSM $\beta 2$ \\
\hline $\begin{array}{l}\text { Dominating } \\
\text { mechanism }\end{array}$ & $\begin{array}{l}\text { Secondary } \\
\text { nucleation }\end{array}$ & $\begin{array}{l}\text { Secondary } \\
\text { nucleation }\end{array}$ & $\begin{array}{l}\text { Secondary } \\
\text { nucleation }\end{array}$ & $\begin{array}{l}\text { Nucleation- } \\
\text { elongation }\end{array}$ \\
\hline MRE & 0.00311 & 0.00138 & 0.00333 & 0.00475 \\
\hline $\mathrm{k}_{+} \mathrm{k}_{\mathrm{n}}\left(\mathrm{M}^{-\mathrm{nc}} \mathrm{h}^{-2}\right)$ & 0.0000698 & 275 & $1.86 \times 10^{18}$ & 48.8 \\
\hline $\mathrm{n}_{\mathrm{c}}(-)$ & 0.00000784 & 0.6 & 3.92 & 0.572 \\
\hline $\mathrm{k}_{+} \mathrm{k}_{2}\left(\mathrm{M}^{-\mathrm{nc}} \mathrm{h}^{-2}\right)$ & 129 & $5.17 \times 10^{6}$ & $4.23 \times 10^{3}$ & - \\
\hline $\mathrm{n}_{2}(-)$ & 0.00166 & 0.123 & 0.2 & - \\
\hline
\end{tabular}

